ISSN 0103-9954

\title{
EFEITO DO TRATAMENTO TÉRMICO SOBRE A RESISTÊNCIA AO CISALHAMENTO DA LINHA DE COLA EM PAINÉIS OSB
}

\section{EFFECT OF THE THERMAL TREATMENT ON THE GLUE-LINE SHEAR STRENGTH OF OSB \\ PANELS}

\author{
Airton Mauro de Lára Santos ${ }^{1}$ Frederico de Souza ${ }^{2}$ Mirian de Almeida Costa ${ }^{3}$ Ricardo Faustino Teles ${ }^{4}$ \\ Valéria Maria Figueiredo Pazetto ${ }^{5}$ Cláudio Henrique Soares Del Menezzi ${ }^{6}$
}

\section{RESUMO}

As pesquisas sobre o tratamento térmico em painéis OSB (oriented strandboard) vêm buscando reduzir a higroscopicidade dos painéis, bem como aliviar as tensões geradas durante o processo de prensagem. Entretanto, o tratamento térmico acima de $160^{\circ} \mathrm{C}$ pode causar inativação da superfície da madeira, tornando-a mais lisa, diminuindo a penetração do adesivo e por conseqüência, reduzindo a qualidade da adesão. O lixamento da superfície de painéis OSB pode tornar a superfície mais rugosa aumentando a qualidade da adesão e a resistência na linha de cola. O presente estudo teve como objetivo avaliar a resistência na linha de cola de painéis OSB tratados termicamente, assim como a influência do lixamento na qualidade da colagem dos painéis. Foram obtidos, aleatoriamente painéis comerciais de OSB que foram tratados termicamente em prensa laboratorial segundo dois níveis temperaturas $\left(190\right.$ e $\left.220^{\circ} \mathrm{C}\right)$, três tempos de duração (12, 16 e 20 minutos). Foram cortados 84 corpos-de-prova (CP) sendo que a metade foi lixada. O ensaio de resistência ao cisalhamento da linha de cola foi conduzido segundo ASTM D1037, sendo avaliado o adesivo resorcinol-formaldeído na gramatura de $360 \mathrm{~g} / \mathrm{m}^{2}$. Os resultados mostraram que o tratamento térmico teve influência na resistência, diminuindo-a levemente em comparação ao material nãotratado. Foi identificado efeito interativo entre a temperatura e o tempo de tratamento. Entretanto, com a operação de lixamento esse efeito foi removido e as diferenças com a testemunha foram diminuídas. Observou-se que os ganhos na resistência com o lixamento foram de até $15 \%$. Sendo assim, o tratamento térmico apresentou pouca influência na resistência da linha de cola e o efeito do lixamento apresentou-se como um processo benéfico, melhorando a qualidade da adesão dos painéis OSB.

Palavras-chave: Painel OSB; tratamento térmico; lixamento; resistência da linha de cola.

\section{ABSTRACT}

Research to improve dimensional stability of oriented strand boards (OSB) through thermal treatment has been done. It aims to reduce the higroscopicity of the wood particles as well as to relieve the compression stresses generated during pressing process. In spite of the advantages, the thermal treatment above $160^{\circ} \mathrm{C}$ can inactive the surface of the wood, reducing the penetration of the adhesive and, this way, reducing the quality of the adhesion. The sanding of the surface of OSB panels can remove this effect, increasing the quality of the adhesion and the glue line strength. The present study aimed to evaluate the effect of the thermal treatment on the glue-line shear strength of OSB panels and the effect of the sanding

1. Engenheiro Florestal, Mestrando do Programa de Pós-graduação em Ciências Florestais, Departamento de Engenharia Florestal, Universidade de Brasília, Caixa Postal 04357, CEP 70919-970, Brasília (DF). airtonmauro@gmail.com

2. Engenheiro Florestal, Mestrando do Programa de Pós-graduação em Ciências Florestais, Departamento de Engenharia Florestal, Universidade de Brasília, Caixa Postal 04357, CEP 70919-970, Brasília (DF). fredericodesouza@hotmail.com

3. Engenheiro Florestal, Mestrando do Programa de Pós-graduação em Ciências Florestais, Departamento de Engenharia Florestal, Universidade de Brasília, Caixa Postal 04357, CEP 70919-970, Brasília (DF). almeida.mira@gmail.com

4. Engenheiro Florestal, Mestrando do Programa de Pós-graduação em Ciências Florestais, Departamento de Engenharia Florestal, Universidade de Brasília, Caixa Postal 04357, CEP 70919-970, Brasília (DF). valeriapazetto@gmail.com

5. Desenhista Industrial, Mestranda do Programa de Pós-graduação em Ciências Florestais, Departamento de Engenharia Florestal, Universidade de Brasília, Caixa Postal 04357, CEP 70919-970, Brasília (DF). rfteles@gmail.com

6. Engenheiro Florestal, Dr., Professor Adjunto do Departamento de Engenharia Florestal, Universidade de Brasília. Caixa Postal 04357, CEP 70919-970, Brasília (DF). cmenezzi@unb.br

Recebido para publicação em 6/02/2008 e aceito em 20/03/2009. 
process on this strength, as well. Commercial OSB were thermally treated according to two temperature levels $\left(190\right.$ and $\left.220^{\circ} \mathrm{C}\right)$, three times $(12,16$ and 20 minutes). Eighty-four samples were cut, both of them were sanded before the gluing (resorcinol-formaldehyde, $360 \mathrm{~g} / \mathrm{m}^{2}$ ). The glue-line shear strength testing was conducted according to ASTM D 1037 standard. The results reveled that the proposed thermal treatment reduced slightly the shear strength The interaction factor between temperature and time on these results was identified. However, sanding process removed this effect and improved significantly shear strength. Therefore, the thermical treatment imparted little effect on the glue-line shear strength, while the sanding process improved the quality of the adhesion in OSB panels.

Keywords: OSB panels; thermal treatment; sanding; glue-line shear strength.

\section{INTRODUÇÃO}

Os produtos engenheirados de madeira (PEM) consistem em uma classe de produtos estruturais que são comumente empregados na construção civil, podendo ser feitos de lâminas ou partículas de madeira (PRION, 2003). Os PEM são utilizados como substitutos de produtos maciços de madeira ou mesmo para substituir aqueles que são produzidos por processos altamente poluentes, antecedidos por agressões ambientais consideráveis para a obtenção da matéria-prima como o aço e o concreto armado, por exemplo. Além disso, os PEM proporcionam uma economia dos materiais utilizados como matéria prima, além de um uso mais eficiente desses produtos. A utilização de madeira reconstituída é uma tendência atual que traz consigo inúmeras vantagens: pré-fabricação, industrialização, homogeneidade, redução da anisotropia e utilização de madeira de rápido crescimento proveniente de florestas plantadas (LIMA et al., 2004). Dentre os principais PEM destaca-se o OSB, o LVL e a madeira laminada colada.

O OSB (Oriented Strand Board) é um painel de partículas de madeira orientadas, finas e longas, conhecidas como "strands", consolidado pelo uso de resinas, calor e pressão. Segundo Tsoumis (1991), esse tipo de painel é tipicamente formado por três camadas, sendo nas externas as partículas de madeira orientadas paralelamente na direção de formação do painel; na interna, a orientação é perpendicular. Podem ser obtidos a partir de madeiras provenientes de desbastes e de troncos finos e tortuosos, bem como de espécies de menor valor comercial. Competem com os compensados, que requerem toras de alta qualidade para a sua manufatura e, por isso, são de custo relativamente superior (WALKER, 2006). É um produto utilizado para aplicações estruturais, como paredes, suportes para forros e pisos, componentes de vigas estruturais, estrutura de móveis, embalagens, entre outras, tendo em vista boas características de resistência mecânica.

Segundo Moslemi (1974), os esforços para a melhoria de estabilidade dimensional de painéis reconstituídos devem ser focados para dois aspectos básicos principais: a redução da higroscopicidade da madeira utilizada e a liberação de tensões impostas durante a prensagem do painel. O tratamento térmico após a produção do painel possibilita o ataque simultâneo a estes dois principais fatores que influenciam a estabilidade dimensional de painéis (DEL MENEZZI, 2004). De acordo com este autor, a madeira pode ser aquecida sob determinadas condições de temperatura, tempo, pressão e umidade, de modo a prover alterações ditas permanentes. Nessas condições, a madeira termicamente tratada pode apresentar alterações de propriedades mecânicas, da estabilidade dimensional e da resistência biológica. No caso específico do painel OSB, o tratamento térmico pós-produção tem melhorado significativamente a estabilidade dimensional como apontam os trabalhos de Okino et al. (2007), Del Menezzi e Tomaselli (2006) e Del Menezzi (2004). Segundo Del Menezzi e Tomaselli (2007) este tipo de tratamento térmico é viável do ponto de vista técnico e econômico, com custo final de US\$ 7,60 a 9,40/ $\mathrm{m}^{3}$, dependendo da temperatura e do tempo de tratamento.

Segundo Kandem et al. (2002), o tratamento térmico ajuda a liberar as tensões da madeira após a remoção da hemicelulose e, em função da degradação térmica, ocorre uma redução na aspereza e escurecimento da superfície das madeiras tratadas. Esta melhora na textura superficial dos painéis tratados é explicada por Christiansen (1990), onde, segundo o autor, no tratamento térmico, ou até mesmo durante a prensagem, se a temperatura excede um limite de aproximadamente $160^{\circ} \mathrm{C}$ ocorrerá a inativação de superfície. Northcott et al. (1959) definiram a inativação de superfície como uma mudança nas condições superficiais da madeira, formando uma espécie de revestimento, que é induzida ou intensificada pelo processo de produção e que dificulta a adesão entre os substratos. Os mesmos autores observaram que entre 
os problemas acarretados pela inativação térmica, a penetração insuficiente da umidade do adesivo na madeira durante o período de cura é um fator limitante no processo de consolidação. Ou seja, a água se concentra na linha de cola, prejudicando a adesão. Com o lixamento da superfície, os problemas causados pela inativação seriam atenuados, uma vez que removendo parte deste revestimento, a textura ficaria mais rugosa, facilitando a penetração do adesivo na madeira, melhorando a qualidade da união colada.

Para testar o desempenho de um adesivo, deve-se submetê-lo a testes de carregamento específico, como de cisalhamento e tração na linha de cola, avaliando a eficiência do adesivo na união das lâminas sob uma determinada temperatura e condições de umidade durante um tempo específico (USDA, 1999). A resistência na linha de cola é uma das propriedades que devem ser consideradas quando se deseja unir painéis de madeira, de modo a obter a resistência necessária para não comprometer a integridade dos PEM's, dando a essa segurança e rigidez a estrutura.

Se comprovada a eficiência do lixamento da superfície da linha de cola em painéis OSB tratados termicamente, estes podem se tornar um produto diferenciado, mais resistente. Afinal, a propriedade física seria melhorada pelo tratamento térmico e a propriedade mecânica seria aumentada com o tratamento da lixa. O seu nicho de utilização poderá se expandir para situações mais severas podendo aumentar mais ainda a sua competitividade com os aglomerados e compensados.

Diante do exposto, o presente trabalho teve como objetivo avaliar a resistência na linha de cola de painéis de OSB tratados termicamente, assim como a influência do lixamento na superfície na qualidade da colagem.

\section{MATERIAL E MÉTODO}

Dois painéis de partículas orientadas (OSB), produzidas com madeira de Pinus sp, com densidade de $0,64 \mathrm{~g} / \mathrm{cm}^{2}$, três camadas na sua formação e dimensões de $244 \times 122 \times 1,25 \mathrm{~cm}$ (largura x comprimento $\mathrm{x}$ espessura), foram adquiridos aleatoriamente no estoque de um fabricante de painéis OSB localizada no município de Ponta Grossa - PR. O adesivo empregado na fabricação dos painéis foi o fenol-formaldeído nas camadas externas e o isocianato na camada interna. Os painéis foram mantidos em uma sala de climatização a uma temperatura de $20 \pm 3^{\circ} \mathrm{C}$ e um teor de umidade de $65 \pm 1 \%$, até atingir massa constante.

Os painéis foram tratados termicamente a partir da metodologia proposta por Del Menezzi (2004), em prensa laboratorial com controle de temperatura e pressão, em dois níveis de temperatura $\left(190^{\circ} \mathrm{C} \mathrm{e}\right.$ $\left.220^{\circ} \mathrm{C}\right)$ e três níveis de tempo $(12,16,20$ minutos) totalizando seis tratamentos mais a testemunha, na qual não foi aplicado nenhum tratamento térmico. Durante o tratamento uma pressão de $0,17 \mathrm{kPa}$ foi aplicada para prover o contato entre os pratos da prensa e o painel.

Os corpos-de-prova (CP) para o ensaio de resistência da linha de cola foram obtidos seguindo os procedimentos da norma ASTM D 1037:99 e possuíam dimensões iguais a 44 x 51 x 12,5 mm (largura, comprimento e espessura). Para atingir a espessura especificada para o ensaio, os CP foram colados dois a dois com resina à base de resorcinol-formaldeído da marca Cascophen RS a uma gramatura de $360 \mathrm{~g} / \mathrm{m}^{2}$. As dimensões finais dos CP foram iguais a 44 x 51 x $25 \mathrm{~mm}$ (largura, comprimento e espessura), de acordo com a Figura 1. Com o intuito de avaliar o efeito do lixamento na resistência da linha de cola, metade dos CPs foi lixado manualmente na face de cisalhamento com uma lixa de granulometria $\mathrm{P} 100$, realizando o movimento em formato de "8" doze vezes por peça. Após, os CP foram colados na face lixada com a mesma resina na mesma gramatura. Os CP foram colados no sentido paralelo às fibras. 


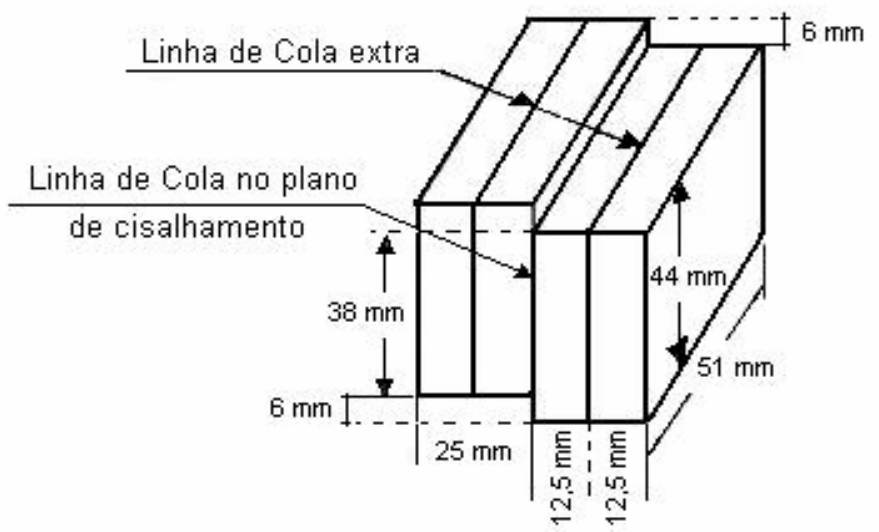

FIGURA 1: Corpo-de-Prova do ensaio de cisalhamento da linha de cola. Fonte: Adaptado de ASTM, 1999.

FIGURE 1: Sample of glue-line shear test. Source: Adapted from ASTM, 1999.

O ensaio de cisalhamento da linha de cola foi realizado em uma maquina universal de ensaio INSTRON segundo norma ASTM D 1037:99, com velocidade de carregamento 0,6 mm/min, até a ruptura. A Tabela 1 apresenta os tratamentos utilizados bem como o número de CP por tratamento. Também foi avaliado o comportamento de corpos-de-prova sem nenhum tratamento térmico, mas com e sem lixamento (testemunhas). Para cada tratamento seis corpos-de-prova (CP) foram cortados, totalizando assim $84 \mathrm{CPs}$.

TABELA 1: Tratamentos utilizados no ensaio de cisalhamento da linha cola de painéis OSB tratados termicamente.

TABLE 1: Treatments applied on glued line shear test of thermically treated OSB panels.

\begin{tabular}{|c|c|c|c|c|}
\hline Tratamento & Temperatura $\left({ }^{\circ} \mathrm{C}\right)$ & Tempo (min) & Lixamento & N. CP \\
\hline \multirow{2}{*}{ Testemunha } & \multirow{2}{*}{0} & \multirow{2}{*}{0} & $\mathrm{~L}$ & 6 \\
\hline & & & NL & 6 \\
\hline \multirow{2}{*}{$\mathrm{T}-1$} & \multirow{6}{*}{190} & \multirow{2}{*}{12} & $\mathrm{~L}$ & 6 \\
\hline & & & NL & 6 \\
\hline \multirow{2}{*}{$\mathrm{T}-2$} & & \multirow{2}{*}{16} & $\mathrm{~L}$ & 6 \\
\hline & & & NL & 6 \\
\hline \multirow{2}{*}{$\mathrm{T}-3$} & & \multirow{2}{*}{20} & $\mathrm{~L}$ & 6 \\
\hline & & & NL & 6 \\
\hline \multirow{2}{*}{$\mathrm{T}-4$} & \multirow{6}{*}{220} & \multirow{2}{*}{12} & $\mathrm{~L}$ & 6 \\
\hline & & & NL & 6 \\
\hline \multirow{2}{*}{$\mathrm{T}-5$} & & \multirow{2}{*}{16} & $\mathrm{~L}$ & 6 \\
\hline & & & NL & 6 \\
\hline \multirow{2}{*}{$T-6$} & & \multirow{2}{*}{20} & $\mathrm{~L}$ & 6 \\
\hline & & & NL & 6 \\
\hline
\end{tabular}

Em que: $\mathrm{L}=\overline{\text { lixado; } \mathrm{NL}=\text { não-lixado; } \mathrm{CP}=\text { corpo-de-prova. }}$

\section{RESULTADOS E DISCUSSÃO}

A Figura 2 apresenta o aspecto geral dos CPs após o ensaio de cisalhamento na linha de cola. A ruptura ocorreu sempre no OSB, não apresentando falha na linha de cola em nenhum dos CPs ensaiados. Desta forma, a resistência da linha de cola foi superior à resistência de cisalhamento do OSB, o que evidencia a boa qualidade da ligação adesiva produzida.

Os resultados obtidos para a resistência ao cisalhamento da linha de cola para todos os tratamentos são apresentados na Figura 3. A seguir serão analisados separadamente o efeito do tratamento térmico proposto (sem lixa) e o efeito do lixamento sobre os resultados obtidos. Os dados discrepantes foram corrigidos através de uma análise de outlier, a qual indicou que três dados mostraram-se discrepantes e foram retirados, sendo um do tratamento 4 não lixado, e dois do tratamento 5 lixado, totalizando, portanto, $81 \mathrm{CPs}$. 

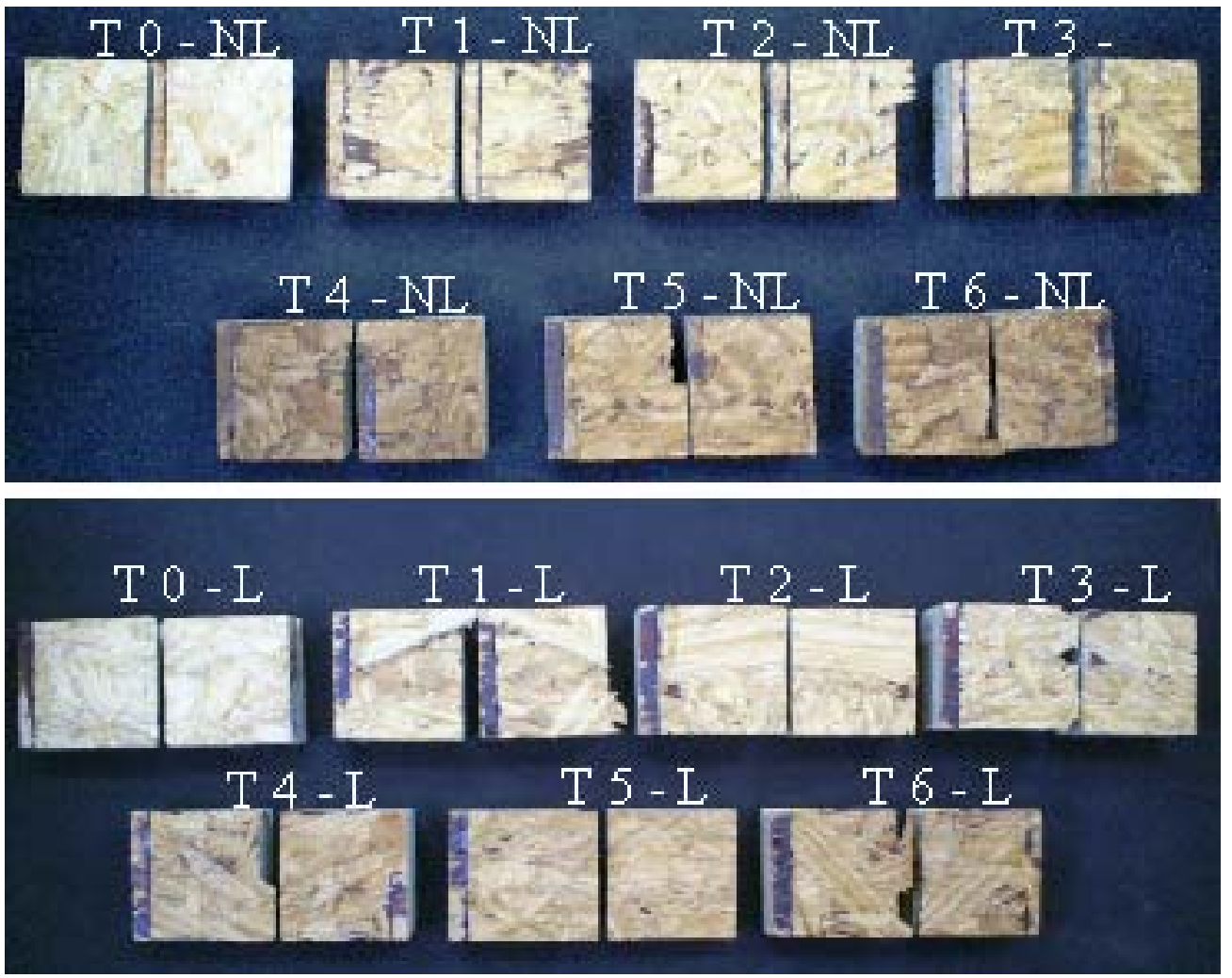

FIGURA 2: Aspecto das amostras após o ensaio de cisalhamento na linha de cola. (NL = não-lixado; $\mathrm{T}=$ tratamento; $\mathrm{L}=$ lixado)

FIGURE 2: Result of the glue-line shear test. Where ( $\mathrm{L}=$ sanded; $\mathrm{T}=$ tratment; $\mathrm{NL}=$ not sanded).

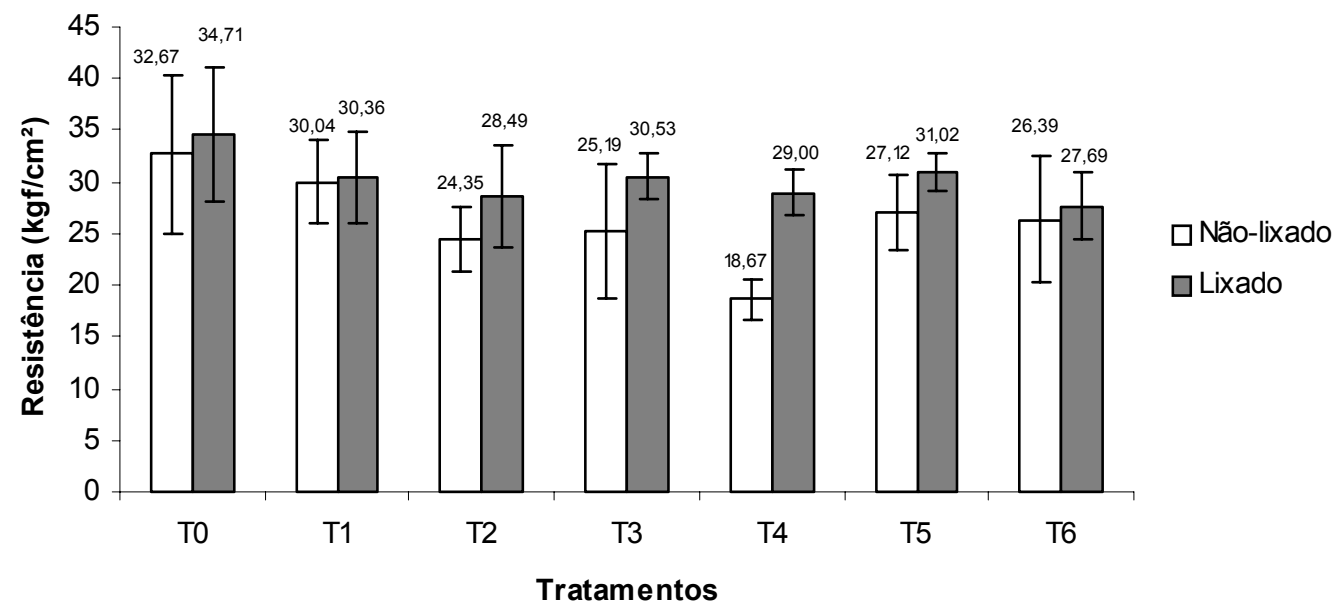

FIGURA 3: Efeito do lixamento na resistência da linha de cola.

FIGURE 3: Sanding effect on the glue-line strength.

\section{Efeito do tratamento térmico}

O resultado do teste de Dunnett é apresentado na Tabela 2. Foi constatado que apenas os tratamentos $\mathrm{T} 2\left(190^{\circ} \mathrm{C} ; 16 \mathrm{~min}\right)$ e $\mathrm{T} 4\left(220^{\circ} \mathrm{C} ; 12 \mathrm{~min}\right)$ diferiram estatisticamente da testemunha, e que os demais apresentaram médias estatisticamente iguais. Ribeiro (2006), avaliando a rugosidade dos painéis tratados termicamente da mesma origem do presente trabalho, observou que esses mesmos tratamentos apresentaram diferenças significativas no tocante à rugosidade da superfície: superfícies mais lisas que a da testemunha para todos os parâmetros de rugosidade analisados (Ra, Rz e Rq). 
TABELA 2: Teste de Dunnett para a resistência na linha de cola com relação ao efeito não-lixado.

TABLE 2: Dunnett's Test of shear in glue-line in relation of not sanded effect.

\begin{tabular}{|c|c|c|c|c|}
\hline Tratamento (I) & Testemunha $(\mathrm{J})$ & Diferenças de Médias (I - J) & Erro Padrão & Significância \\
\hline T1 & T0 & $-2,63$ & 2,991 & 0,888 \\
\hline $\mathrm{T} 2$ & T0 & $-8,32 *$ & 2,991 & 0,042 \\
\hline $\mathrm{T} 3$ & T0 & $-7,48$ & 2,991 & 0,078 \\
\hline $\mathrm{T} 4$ & T0 & $-14,00 *$ & 3,137 & 0,000 \\
\hline T5 & T0 & $-5,55$ & 2,991 & 0,278 \\
\hline T6 & T0 & $-6,28$ & 2,991 & 0,178 \\
\hline
\end{tabular}

Em que: * = Difere estatisticamente ao nível de 5\% pelo Teste de Dunnett.

Este resultado pode ter influenciado na qualidade da adesão dos CPs, diminuindo a penetração do adesivo e a resistência na linha de cola. Segundo Frihart (2005), para que qualquer ligação adesiva seja formada é necessário o contato em nível molecular, e assim o adesivo deve fluir pelos poros da superfície que formam a rugosidade, os quais estão presentes em quase todos os materiais. Salienta ainda, que as ligações mecânicas formadas pelo adesivo (ganchos) podem ser menos resistentes caso os poros sejam pequenos, isto é, o material apresente baixa rugosidade. Deve ser observado, ainda, que esses ganchos são mais importantes para prover resistência maior ao esforço tangencial (cisalhamento) em comparação aos esforços normais (compressão e tração). River et al. (1991) afirmam que até certo ponto a resistência de juntas coladas aumenta com o aumento da porosidade. Isso porque, há uma área maior de contato para formação das ligações adesivas, bem como a formação de ganchos mecânicos é melhorada em função da maior porosidade. De fato, Christiansen (1990) afirma que o fechamento dos microporos é um dos três mecanismos físicos envolvidos na inativação térmica da superfície. Os outros dois são: a migração de extrativos para superfície e a reorientação molecular das microfibilas.

A Tabela 3 apresenta o resultado da ANOVA fatorial para se avaliar o efeito da temperatura e do tempo do tratamento térmico. Observa-se que não foi identificado efeito isolado nem da temperatura e nem do tempo, mas sim uma interação entre esses fatores. Desta forma, o efeito da temperatura vai depender do tempo do tratamento.

TABELA 3: Análise da temperatura e do tempo dentro do grupo não-lixado.

TABLE 3: Temperature and time analysis in the not sanded group.

\begin{tabular}{lccc|c|c|c}
\hline Fonte de Variação & $\begin{array}{c}\text { Graus de } \\
\text { Liberdade }\end{array}$ & $\begin{array}{c}\text { Soma de } \\
\text { Quadrados }\end{array}$ & $\begin{array}{c}\text { Quadrado } \\
\text { Médio }\end{array}$ & F calculado & Significância \\
\hline Temperatura & 1 & 53,05 & 53,05 & 2,507 & 0,124 \\
Tempo & 2 & 14,83 & 7,41 & 0,351 & 0,707 \\
Temperatura* & 2 & 338,44 & 169,22 & 7,996 & $0,002^{*}$ \\
Tempo & 29 & 613,70 & 21,16 & & \\
Erro & 35 & 23723,54 & & & \\
\hline Total & &
\end{tabular}

Em que: * = Difere estatisticamente ao nível de $\alpha=5 \%$.

A interação entre os fatores era esperada, uma vez que a ocorrência da inativação de superfície influencia diretamente a qualidade da adesão dos painéis tratados termicamente (CHRISTIANSEN, 1990; KANDEM et al., 2002) A Figura 4 ilustra a interação entre os fatores temperatura e tempo. Evidencia-se que tratamentos à $220^{\circ} \mathrm{C}$ são beneficiados quando são mais prolongados, e os valores de resistência são aumentados; enquanto que à $190^{\circ} \mathrm{C}$ os valores são diminuídos. 


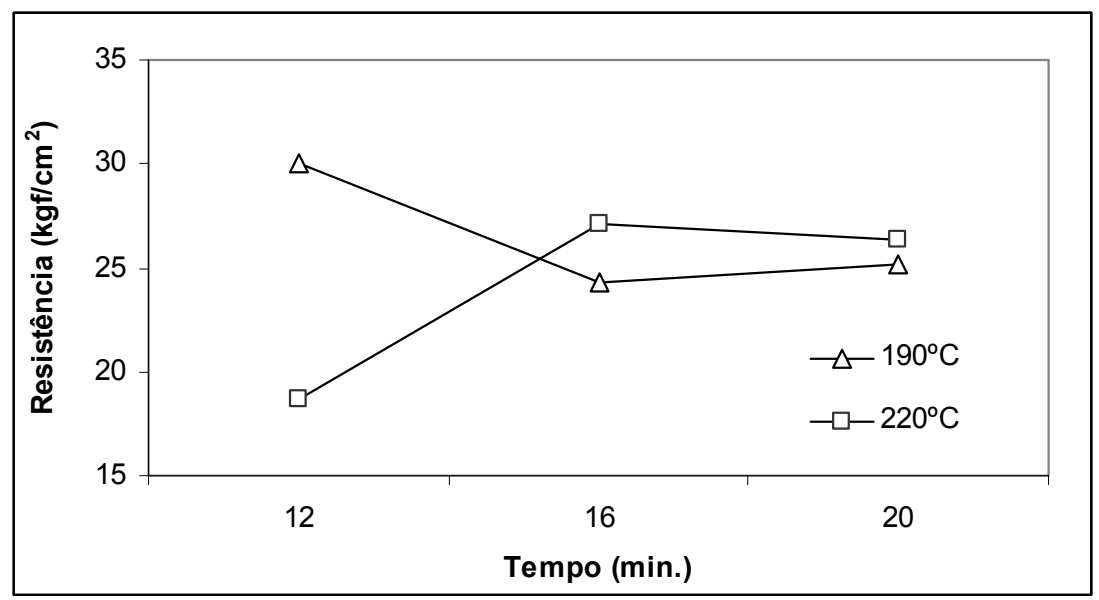

FIGURA 4: Efeito da temperatura e do tempo de tratamento térmico sobre a resistência ao cisalhamento da linha de cola de painéis OSB.

FIGURE 4: Effect of the temperature and time on the glue-line shear strength of OSB panels.

A literatura relata que a degradação térmica de painéis OSB é mais pronunciada em temperaturas mais elevadas (DEL MENEZZI E TOMASELLI, 2006; DEL MENEZZI, 2004). Esse resultado não era esperado haja vista que o escurecimento da superfície do OSB foi mais pronunciado à $220^{\circ} \mathrm{C}$ do que a $190^{\circ} \mathrm{C}$. Esse escurecimento é devido à pirólise mais intensa na superfície do painel, o que leva à produção de furfural, que é um menos higroscópico que os demais polímeros da madeira, o que dificultaria a formação de ligações químicas entre o adesivo e a madeira.

Os resultados apresentados aqui permitem afirmar que o efeito do tratamento térmico sobre a resistência ao cisalhamento da linha de cola foi relativamente pequeno. Alguns estudos têm apresentado resultados similares. Pincelli et al. (2002) aplicaram a retificação térmica $\left(120^{\circ} \mathrm{C}, 140^{\circ} \mathrm{C}, 160^{\circ} \mathrm{C}\right.$ e $\left.180^{\circ} \mathrm{C}\right) \mathrm{em}$ madeiras de Eucalyptus saligna e Pinus caribeae var. hondurensis. Observou-se certa redução na resistência para os três adesivos testados em comparação à testemunha, mas cuja magnitude não foi estatisticamente significativa. Da mesma forma como no presente trabalho, observaram maior porcentagem de ruptura na madeira, que na linha de cola, indicando assim que o tratamento não afetou a adesão produzida. Resultados obtidos por Follrich et al. (2006) para madeira de Picea abies tratada termicamente $\left(200^{\circ} \mathrm{C}\right.$, durante $5,30 \mathrm{e}$ 60 min.) também não apontaram efeito do tratamento sobre a resistência e a carga máxima de cisalhamento em comparação ao material não-tratado.

\section{Efeito do lixamento}

A Tabela 4 apresenta o resultado do teste de Dunnett para os painéis lixados. Observa-se, que apenas T6 apresentou diferença significativa em comparação à testemunha. Nenhum dos demais painéis tratados apresentou diferença significativa. Assim, o lixamento da superfície do material diminuiu o efeito do tratamento térmico, melhorando a penetração do adesivo e diretamente, a resistência ao cisalhamento na linha de cola.

TABELA 4: Teste de Dunnett para a resistência na linha de cola com relação ao efeito lixado.

TABLE 4: Dunnett's Test of shear in glue-line in relation to sanded effect.

\begin{tabular}{cccccc}
\hline Tratamento (I) & Testemunha (J) & Diferenças de Médias (I - J) & Erro Padrão & Significância \\
\hline 1 & 0 & $-4,35$ & 2,337 & 0,278 \\
2 & 0 & $-6,22$ & 2,337 & 0,056 \\
3 & 0 & $-4,18$ & 2,337 & 0,314 \\
4 & 0 & $-5,71$ & 2,337 & 0,091 \\
5 & 0 & $-3,69$ & 2,613 & 0,546 \\
6 & 0 & $-7,01^{*}$ & 2,337 & 0,025 \\
\hline
\end{tabular}

Em que: * = Difere estatisticamente ao nível de 5\% pelo Teste de Dunnett.

Desta forma, a operação de lixamento foi benéfica no sentido de remover o efeito deletério do tratamento térmico, dado que T2 e T4 passaram a apresentar valores estatisticamente iguais à da testemunha. 
Comparando-se as diferenças entre as médias dos tratados e da testemunha (Tabelas 2 e 4), observa-se que essa diferença é menor quando foi aplicado o lixamento, exceto para T1 e T6. Como pode ser observado na Figura 3, o lixamento promoveu também o aumento da resistência nos painéis testemunha, e assim, embora T6 tenha sido também beneficiado a sua diferença em relação à testemunha aumentou para um nível estatisticamente significante. Entretanto, para T1 esse aumento na diferença não foi suficiente para que fosse significativa do ponto de vista estatístico.

Analisando isoladamente a interação dos fatores para o material não-lixados e lixados (Tabelas 3 e 5), nota-se que a interação somente é significativa para o não-lixado, ou seja, os efeitos do tempo e da temperatura do tratamento térmico diretamente na resistência na linha de cola. Entretanto, com o lixamento da superfície ocorre uma melhora da resistência, eliminando o efeito do tratamento térmico, e assim tornando interação não-siginificativa.

TABELA 5: Análise da temperatura e do tempo dentro do grupo lixado.

TABLE 5: Temperature and time analysis in the sanded group.

\begin{tabular}{lcccc|c|c}
\hline Fonte de Variação & $\begin{array}{c}\text { Graus de } \\
\text { Liberdade }\end{array}$ & $\begin{array}{c}\text { Soma de } \\
\text { Quadrados }\end{array}$ & $\begin{array}{c}\text { Quadrado } \\
\text { Médio }\end{array}$ & F calculado & Significância \\
\hline Temperatura & 1 & 2,54 & 2,54 & 0,216 & 0,646 \\
Tempo & 2 & 2,81 & 1,41 & 0,119 & 0,888 \\
Temperatura * Tempo & 2 & 39,91 & 19,95 & 1,694 & 0,202 \\
Erro & 28 & 329,90 & 11,78 & & \\
\hline Total & 34 & 29816,26 & & & \\
\hline
\end{tabular}

Em que: $*=$ Difere estatisticamente ao nível de $\alpha=5 \%$.

A Figura 5 apresenta o efeito da temperatura e do tempo de tratamento térmico sobre a resistência dos CPs lixados. Observa-se que, diferentemente do observado para o material não-lixado (Figura 4), não é possível identificar qualquer tendência nesses dados, o que indica que a operação de lixamento removeu o efeito deletério do tratamento térmico. Desta forma, tanto a temperatura quanto o tempo não tiveram efeito sobre a resistência após o lixamento. Desta forma, acredita-se que o efeito benéfico do lixamento observado nesse trabalho pode ter sido em função de dois fatores: remoção dos extrativos que migraram para a superfície em função do tratamento térmico e aumento dos microporos da superfície liberando assim o caminho para a penetração do adesivo no material. Sendo assim, a operação de lixamento atuou sobre dois dos três mecanismos físicos da inativação térmica da superfície citados por Frihart (2005).

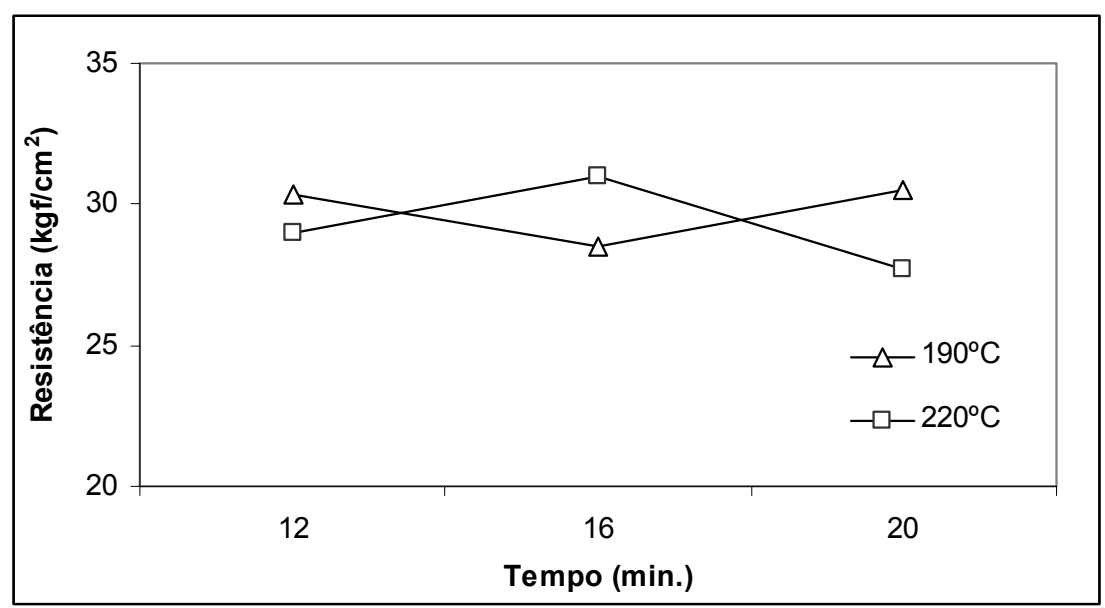

FIGURA 5: Efeito da temperatura e do tempo de tratamento térmico sobre a resistência ao cisalhamento da linha de cola de painéis OSB lixados.

FIGURE 5: Effect of the temperature and time on the glue-line shear strength of sanded OSB panels.

O resultado da análise de variância (ANOVA) para o efeito do lixamento é apresentado na Tabela 7. Observa-se que o material lixado apresentou resistência estatisticamente superior à do material não-lixado. Os ganhos de resistência com lixamento para os tratamentos foram: T0, 6,2\%; T1, 1,1\%; T2, 17,1\%; T3, 21,$2 ; \mathrm{T} 4,55,4 \%$; T5, 13,7\%; e T6, 4,9\%. Na média esse ganho para o material lixado foi de $14,7 \%$. 
TABELA 7: ANOVA e médias para o efeito do lixamento sobre a resistência ao cisalhamento da linha de cola.

TABLE 7: ANOVA and means of the sanding effect on the glue-line shear strength.

\begin{tabular}{l|c|c|c|c|c|c}
\hline Fonte de Variação & Média $\left(\mathrm{kgf} / \mathrm{cm}^{2}\right)$ & $\begin{array}{c}\text { Graus de } \\
\text { Liberdade }\end{array}$ & $\begin{array}{c}\text { Soma de } \\
\text { Quadrados }\end{array}$ & $\begin{array}{c}\text { Quadrado } \\
\text { Médio }\end{array}$ & $\begin{array}{c}\mathrm{F} \\
\text { calculado }\end{array}$ & Significância \\
\hline Lixamento & 1 & 268,45 & 268,45 & 13,063 & 0,001 \\
& $30,25(\mathrm{~L})$ & & & & & \\
Erro & $26,34(\mathrm{NL})$ & 67 & 1376,81 & 20,55 & & \\
\hline Total & 68 & 1645,26 & & & \\
\hline
\end{tabular}

Em que: $\mathrm{L}=$ lixado; NL: = não-lixado.

River et al. (1984) discutem os efeitos do lixamento sobre a qualidade da colagem. Argumentam que um lixamento severo não é recomendado na preparação de superfícies para a colagem, uma vez que danifica as fibras, levando assim à redução da resistência. Entretanto, um lixamento leve, como o feito nesse trabalho, implica na melhoria na umectação pelo adesivo em superfícies que foram inativadas em função da secagem excessiva ou acumulação de extrativos. Belfas et al. (1993) estudaram o efeito do lixamento sobre resistência ao cisalhamento da linha de cola em duas espécies de eucalipto (E. diversicolor e E. marginata) para o adesivo resorcional-formaldeído. Os autores observaram que o lixamento aumentou a umectação da madeira pelo adesivo, refletindo assim no aumento da resistência.

\section{CONCLUSÕES}

O tratamento térmico apresentou pequeno efeito na resistência da linha de cola de painéis OSB, diminuindo-a. Esse efeito dependeu conjuntamente da temperatura e do tempo de tratamento térmico empregados. No entanto, a operação de lixamento removeu esse efeito, bem como aumentou a resistência ao cisalhamento da linha de cola de painéis OSB em até $15 \%$. Assim, recomenda-se o lixamento superficial dos painéis OSB tratados termicamente, como método benéfico que melhor a qualidade da adesão.

\section{REFERÊNCIAS BIBLIOGRÁFICAS}

AMERICAN SOCIETY FOR TESTING AND MATERIALS. ASTM D 1037-Standard test methods of evaluating properties of wood-based fiber and particle panel materials. Philadelphia: PA, 1999.

BELFAS, J. GROVES, K. W, EVANS, P. D. Bonding surface-modified karri and jarrah with resorcinol formaldehyde. I .The effect of sanding on wettability and shear strength. Holz-als-Roh-und-Werkstoff; Berlin, v. 51, n. 4, p. 253259. Apr. 1993.

CHRISTIANSEN, A.W. How overdrying wood reduces its bonding to phenol-formaldehyde adhesives: a critical review of the literature. Part I. physical responses. Wood and Fiber Science, Madison, v. 22, p.441-459, 1990.

DEL MENEZZI, C. H. S. Estabilização dimensional por meio do tratamento térmico e seus efeitos sobre as propriedades de painéis de partículas orientadas (OSB). 2004. 226 f. Tese (Doutorado em Engenharia Florestal). Setor de Ciências Agrárias, Universidade Federal do Paraná.

DEL MENEZZI, C. H. S., TOMASELLI, I. Technological and economic feasibility to produce OSB with enhanced properties in Brazil In: INTERNATIONAL PANEL PRODUCTS SYMPOSIUM, 2007, Cardiff. Proceedings... Bangor: BioComposites Centre, 2007. p. 35 - 45. v.1

DEL MENEZZI, C. H. S., TOMASELLI, I. Contact thermal post-treatment of oriented strandboard: a preliminary study. Holz Als Roh Und Werkstoff, Berlin, v. 64, n. 3, p. 212 - 217, Mar. 2006.

FOLLRICH, J., MULLER, U., GINDL, W. Effects of thermal modification on the adhesion between spruce wood (Picea abies Karst.) and a thermoplastic polymer. Holz-als-Roh-und-Werkstoff; Berlin, v. 64, n. 5 p.373-376. May 2006.

FRIHART, C. R. Wood Adhesion and Adhesives. In: ROWEL, R. Wood Chemistry and Wood Composites. New York: CRC Press, 2005.

FOREST PRODUCTS LABORATORY. Wood Handbook: wood as an engineering material. Madison: Department of Agriculture, 1999. $463 \mathrm{p}$.

KANDEM, D. P; PIZZI, A..; JERMANNAUD, A. Durability of heat-treated wood. Holz als Roh-und Werkstoff. Springer-Verlag. 2002. 6 p.

MOSLEMI, A. A. Particleboard: Materials. Carbondale: Southern Illinois University Press, 1974. 244 p. v.1. 
NORTHCOTT, P.L et al. Undercure: casehardening in plywood. Forest Products Journal, Madison, v. 9, n. 12, p. 442-451, Sept. 1959.

OKINO, E. Y.A., TEIXEIRA, D. E., DEL MENEZZI, C. H. S. Post-thermal treatment of oriented strandboard (OSB) made from cypress (Cupressus glauca Lam.). Maderas. Ciencia y Tecnología ,Concepcion, v. 9, n. 3, p.199 - 210 , mar. 2007.

PINCELLI, A. L. P. S. M.; BRITO, J. O., CORRENTE, J. E. Avaliação da termorretificação sobre a colagem de Eucalyptus saligna e Pinus caribaea var. hondurensis. Scientia Forestalis, n. 61, p. 122-132, jun. 2002.

PRION, H. G. L. Engineering wood products for structural purpose. In: THELANDERSSON, S.; LARSEN, H. J. Timber Engineering. London: Jonh Wiley and Sons, 2003

RIBEIRO, R. B. Avaliação da resistência de ligação e da qualidade da superfície de painéis OSB tratados termicamente. 2006. 28 f. Trabalho de conclusão de curso. Departamento de Engenharia Florestal, Universidade de Brasília.

RIVER, B. R; VICK, C.B.; GILLISPIE, R. H. Wood as an Adherent. New York : Marcel Dekker, 1991. 238 p.

SELLERS, T. Wood adhesive: innovations and applications in North America. Forest Products Journal, Madison, v. 51, n. 6, Jun. p. 12-22, 2001.

TSOUMIS, G. Science and technology of wood: structure, properties, utilization. New York: Chapman \& Hall, 1991. p. 309-339.

WALKER, J. C .F. Primary wood processing: principles and practice. Amsterdam: Springer-Verlag, 2006.602 p. 